\title{
Hemothorax and Hemorrhagic Ascites: A Rare Presentation of Endometriosis
}

Harry Wang, MD

\section{INTRODUCTION}

Endometriosis is a common diagnosis in reproductive age women characterized by the presence of endometrial tissue outside the uterine cavity. ${ }^{1}$ Although it is typically confined to the pelvis, rare manifestations involving the thorax, abdomen or pericardium have been documented. ${ }^{2-5}$ Even more rare is the concurrent presence of endometriosis-associated hemorrhagic pleural effusion and ascites, which can prove a diagnostic challenge. We describe a case of hemothorax and hemorrhagic ascites in a woman with shortness of breath and abdominal pain.

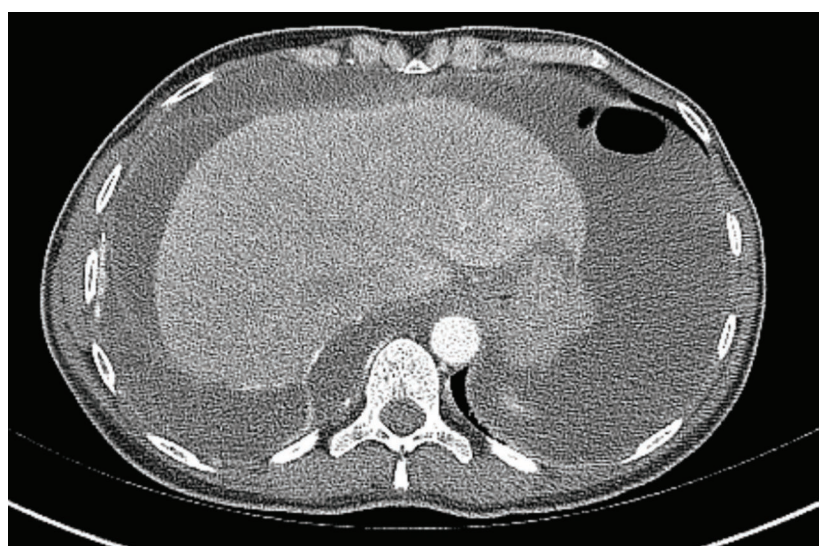

Figure 1. CT imaging showing moderate simple ascites throughout the abdomen.

\section{CASE PRESENTATION}

A 41-year-old woman with a history of bilateral spontaneous pneumothoraces (4-5 years ago), congenital hearing loss, anemia, and peptic ulcer disease presented to the ED with several days of pleuritic chest pain, dyspnea on exertion, and abdominal pain. She denied fevers, chills, night sweats, cough, hemoptysis, vaginal bleeding, or pelvic pain, but did endorse a 14-pound unintentional weight loss over the past several months with no change in appetite. Her last menstrual period was exactly 1 month prior to presentation. On exam, patient had a temperature of 99.6F, blood pressure of $92 / 54 \mathrm{mmHg}$, heart rate of $83 \mathrm{bpm}$, and oxygen saturation of $99 \%$ on room air. She was thin-appearing with a distended abdomen that was diffusely tender to palpation. There were decreased breath sounds in her right lower lung field, but she was clear to auscultation on the left side. Initial laboratory studies were only notable for microcytic anemia (hemoglobin $12.3 \mathrm{~g} /$ $\mathrm{dL}$ ) and elevated CRP (18.4 mg/dL). Her basic metabolic panel and complete blood count were otherwise within normal limits. Chest and abdominal computed tomography (CT) imaging revealed a large loculated right-sided pleural effusion, large-volume ascites (Figure 1) without cirrhosis and a $5 \mathrm{~cm}$ multicystic right adnexal mass (Figure 2). Thoracentesis and paracentesis were performed, both yielding grossly bloody fluid. Cultures and gram and acid-fast stains of both fluid samples revealed no organisms, and cytology was negative for malignancy; HIV and hepatitis serologies were negative and the serum-ascites albumin gradient was less than 1.0. A right-sided chest tube was placed to facilitate further drainage (Figure 3); this was subsequently removed after several days of minimal drainage. A transvaginal pelvic ultrasound revealed a cystic right ovarian mass with thickened, nodular internal septations. CA 125 was mildly elevated, but CEA and CA 19-9 were normal.

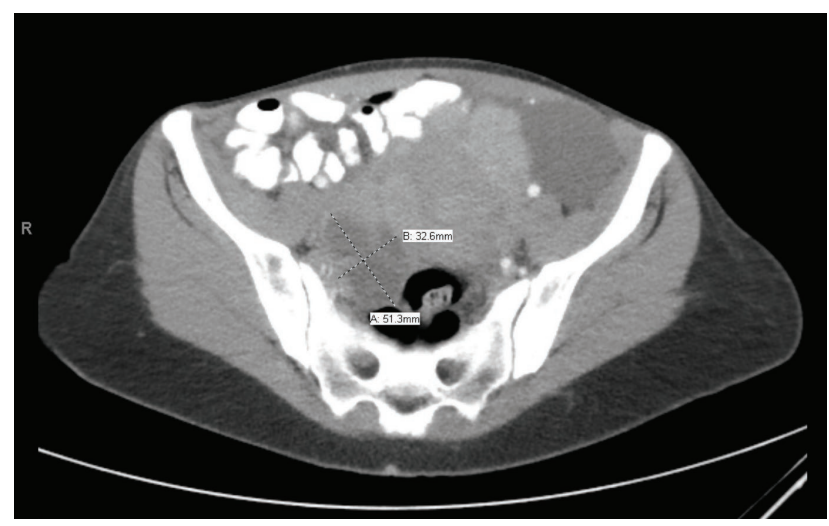

Figure 2. CT imaging demonstrating a multicystic $5.1 \mathrm{~cm}$ right adnexal mass

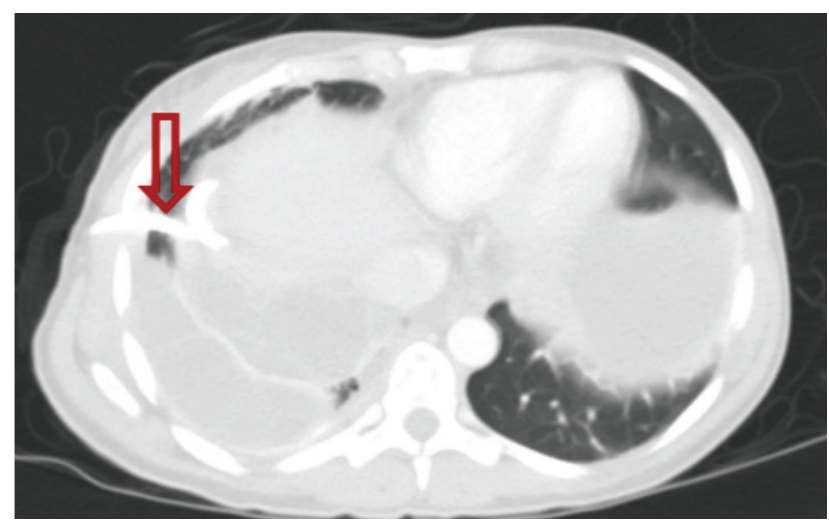

Figure 3. CT imaging showing a right mediastinal loculated fluid collection with a right-sided chest tube in place (arrow). 


\section{DIFFERENTIAL DIAGNOSIS}

Mr. M suffered discrete episodes of altered mental status that were precipitated by anesthesia, sleep deprivation due to late lab draws, nighttime administration of ciprofloxacin and baclofen, and known triggers of his post-traumatic stress disorder (including hospital helicopter landings and thirst). These episodes decreased in frequency with adequate sleep, reorientation measures, control of his thirst, use of atypical antipsychotics, and removal of a fecal management system. His ammonia was elevated initially at 49, at which point he was empirically started on lactulose and rifaximin with stool output at or above goal. He was euglycemic throughout his stay. The patient had no kidney disease or elevated BUN to suggest uremia. Exhaustive brain imaging did not reveal any significant intracranial pathology. Comprehensive infectious workup found no new infection or recurrence of his previous joint infection. Given his critical illness, waxing and waning mental status, and lack of improvement despite correction of other identifiable causes, the diagnosis of delirium was made.

\section{DIFFERENTIAL DIAGNOSIS}

The differential for bloody pleural effusion and ascites includes a variety of infectious and inflammatory etiologies. Her history of bilateral spontaneous pneumothoraces and congenital hearing loss could suggest an autoimmune or connective tissue disorder; anti-nuclear antibody, rheumatoid factor, anti-glomerular basement membrane antibody, and anti-neutrophil cytoplasmic antibody were negative. She was appropriately worked up for HIV, viral hepatitis and tuberculosis, and cultures were unrevealing. She had no signs of cirrhosis or portal hypertension on imaging. A prominent finding on abdominal imaging was the ovarian mass, as that could point toward malignancy or ruptured hemorrhagic ovarian cyst. In the setting of both pleural effusion and ascites, the presence of an ovarian tumor completes the triad of findings for Meigs syndrome, a relatively benign condition in which both fluid collections resolve after resection of the tumor.

\section{OUTCOME AND FOLLOW UP}

Given the concern for malignancy or Meigs syndrome, patient underwent diagnostic laparoscopy in which 850 cc of bloody ascites was evacuated; during this procedure, extensive endometriosis was noted on the peritoneal surfaces with bilateral hydrosalpinx. No active source of bleeding was identified. Both ovaries were devoid of endometriotic implants, so the planned adnexal mass resection was aborted. Post-operatively, her dyspnea and abdominal pain improved, and she was able to be discharged in two days with gynecology follow-up. The patient presented for clinical follow-up with her gynecologist 2 weeks after discharge. She reported minimal pain, normal bowel and bladder function and could tolerate a regular diet. Pathology from the evacuation revealed no malignancy, and medical and surgical (adnexal resection) options were discussed. The patient opted for observation only, as she desired to have another child in the future.

\section{DISCUSSION}

This case illustrates the importance of suspecting atypical presentations of endometriosis. The simultaneous presence of hemothorax and hemoperitoneum suggests a communication via diaphragmatic pores, a similar mechanism to that of hepatic hydrothorax. ${ }^{2}$ Given this patient's presentation and imaging findings, infection and malignancy were aggressively and appropriately worked up, but it is crucial for internists not to omit this relatively common diagnosis from the differential, especially since the management is typically uncomplicated. Meigs syndrome was also an important consideration, as it is characterized by the triad of benign ovarian tumor, pleural effusion and ascites; fluid collections typically resolve after tumor resection in Meigs syndrome. ${ }^{6}$ Treatment options for endometriosis range from simple observation to medical therapy with leuprolide or nonsteroidal anti-inflammatories. For patients with bloody pleural effusions and ascites refractory to medical management, more definitive therapy may involve hysterectomy with salpingo-oophorectomy. ${ }^{1}$

\section{KEY POINTS}

- Hemorrhagic ascites and hemothorax are uncommon presentations of endometriosis that require extensive initial evaluation for infection and malignancy.

- Meigs syndrome should be considered in young women presenting with the triad of benign ovarian tumor, pleural effusion and ascites

- Treatment for endometriosis can vary from observation, NSAID therapy to surgical evacuation and hysterectomy with salpingo-oophorectomy.

\section{REFERENCES}

1. Wee-Stekly, Wei-Wei, Cynthia Chia Yng Kew, and Bernard Su Min Chern. Endometriosis: a review of the diagnosis and pain management. Gynecology and Minimally Invasive Therapy. 2015; 4: 106-109.

2. Lee, H.T., Wang, H.C., Huang, I.T., Chang, H.C. and Lu, J.Y. Endometriosis associated with hemothorax. Journal of the Chinese Medical Association, 2006; 69(1): $42-46$

3. Rawala, M. S. et al. A rare case of cyclical hemothorax: thoracic endometriosis syndrome. Case Reports in Pulmonology. 2018: 1-4.

4. Tiwari, Abhinav, Himani Sharma, Hermann Simo, and M. S. Khan. An uncommon cause of hemopneumothorax In a young female: a case report. Am J Respir Crit Care Med. 2017; 195: A3526.

5. Varun N, Tanwar R. A Rare Presentation of Endometriosis with Massive Haemorrhagic Ascites: A Case Report. Gynecol Obstet Case Rep. 2016; 3:1.

6. Nemeth, A. J. \& Patel, S. K. Meigs Syndrome Revisited. Journal of Thoracic Imaging. 2003; 18: 100-103. 